\title{
COMPLETE REDUCIBILITY AND SEPARABLE FIELD EXTENSIONS
}

\author{
MICHAEL BATE, BENJAMIN MARTIN, AND GERHARD RÖHRLE
}

\begin{abstract}
Let $G$ be a connected reductive linear algebraic group. The aim of this note is to settle a question of J-P. Serre concerning the behaviour of his notion of $G$-complete reducibility under separable field extensions. Part of our proof relies on the recently established Tits Centre Conjecture for the spherical building of the reductive group $G$.
\end{abstract}

\section{INTRODUCTION}

Throughout, $G$ denotes a connected reductive linear algebraic group defined over a field $k$. Following Serre, [13, a subgroup $H$ of $G$ is called $G$-completely reducible over $k$ ( $G$-cr over $k$ ) if whenever $H$ is contained in a $k$-defined parabolic subgroup $P$ of $G$, there exists a $k$-defined Levi subgroup of $P$ containing $H$. In case $V$ is a finite dimensional $k$ vector space and $G=\mathrm{GL}(V)$, a subgroup $H$ of $G$ is $G$-completely reducible over $k$ precisely when $V$ is a semisimple $H$-module, [13, 1.3, 3.2.2]. In this sense, Serre's notion generalizes the usual concept of complete reducibility in representation theory. For more details and further results on this notion, see [12], [13], [1], [3], and [4].

The following theorem answers a question of Serre.

Theorem 1.1. Suppose $k_{1} / k$ is a separable extension of fields. Let $G$ be a reductive group defined over $k$, and let $H$ be a $k$-defined subgroup of $G$. Then $H$ is $G$-completely reducible over $k$ if and only if $H$ is $G$-completely reducible over $k_{1}$.

The reverse implication in Theorem 1.1 is proved in [4, Thm. 5.11]. The proof of [4, Thm. 5.11] rests on a general rationality result, [4, Thm. 3.1], concerning $G$-orbits in an affine variety. We present a proof of the forward direction of the statement in Section 3 based on the recently established Tits Centre Conjecture, Theorem 2.3.

2010 Mathematics Subject Classification. 51E24, 20E42, $20 \mathrm{G} 15$.

Key words and phrases. G-complete reducibility, spherical buildings, Tits Centre Conjecture. 
Remarks 1.2. (i). In [4, Ex. 5.12], we showed that Theorem 1.1 holds when $G=\mathrm{GL}(V)$.

(ii). Theorem 1.1 was proved in [1, Thm. 5.8] for $k$ perfect, by passing back and forth between $k$ and its algebraic closure $\bar{k}$ and between $k_{1}$

and $\bar{k}$. In general this approach fails, because the extension $\bar{k} / k$ need not be separable.

(iii). There are examples showing that each implication in Theorem 1.1 fails without the separability assumption on the extension $k_{1} / k$; see [1, Ex. 5.11] and [3, Ex. 7.22].

\section{The Centre Conjecture for spherical Buildings}

Let $\Delta_{k}$ denote the spherical building of $G$ over $k$, [15, Sec. 5]: the simplices of $\Delta_{k}$ correspond to $k$-defined parabolic subgroups of $G$. Given a $k$-defined parabolic subgroup $P$ of $G$, we denote the simplex corresponding to $P$ in $\Delta_{k}$ by $\sigma_{P}$. Throughout, we identify $\Delta_{k}$ with its geometric realization, which is a bouquet of spheres [13].

An apartment in $\Delta_{k}$ consists of the simplices $\sigma_{P}$ corresponding to all $k$-defined parabolic subgroups $P$ of $G$ which contain a fixed maximal $k$-split torus of $G$; it is a subcomplex whose geometric realization is a sphere. Any two points of $\Delta_{k}$ lie in a common apartment. We say that $x, y \in \Delta_{k}$ are opposite if they are opposite in some apartment that contains them both. It can be shown that if $x$ and $y$ are opposite in some apartment that contains them both, then they are opposite in any apartment that contains them both. If $x, y \in \Delta_{k}$ are not opposite, then there is a unique geodesic joining them, [13, §2.1.4]. Two simplices $\sigma_{P}$ and $\sigma_{Q}$ are said to be opposite if every point of $\sigma_{P}$ is opposite a point of $\sigma_{Q},[13, \S 2.1 .4]$. In terms of parabolic subgroups of $G$, the simplices $\sigma_{P}$ and $\sigma_{Q}$ corresponding to $k$-defined parabolic subgroups $P$ and $Q$ of $G$ are opposite in $\Delta_{k}$ if and only if $P \cap Q$ is a common Levi subgroup of $P$ and $Q$ (this Levi subgroup is then automatically $k$-defined).

Let $\Sigma$ be a subcomplex of $\Delta_{k}$. We say that $\Sigma$ is convex if whenever $x, y \in \Sigma$ are not opposite, then $\Sigma$ contains the geodesic between $x$ and $y,[13, \S 2.1]$.

Suppose $\Sigma$ is a convex subcomplex of $\Delta_{k}$. Serre has shown that $\Sigma$ is contractible — that is, $\Sigma$ has the homotopy type of a point — if and only if there exists a point of $\Sigma$ which has no opposite in $\Sigma$; see [13, $\S 2.2]$. The following terminology is due to Serre [13, Def. 2.2.1]:

Definition 2.1. Let $\Sigma$ be a convex subcomplex of $\Delta_{k}$. We say that $\Sigma$ is $\Delta_{k}$-completely reducible (or $\Delta_{k}$-cr) if every simplex in $\Sigma$ has an opposite in $\Sigma$. 
Serre has shown that the group-theoretic definition of $G$-complete reducibility over $k$ has the following building-theoretic interpretation, [13]: Given a subgroup $H$ of $G$, let

$$
\Delta_{k}^{H}=\left\{\sigma_{P} \mid P \text { is a } k \text {-defined parabolic subgroup containing } H\right\} .
$$

Then $\Delta_{k}^{H}$ is a convex subcomplex of $\Delta_{k}$ ([13, Prop. 3.1]), the fixed point subcomplex of $\Delta_{k}$ under the action of $H$, and $H$ is $G$-completely reducible over $k$ if and only if $\Delta_{k}^{H}$ is $\Delta_{k}$-cr, [13, 2.3.1, 3.2]. Equivalently, $H$ is not $G$-completely reducible over $k$ if and only if $\Delta_{k}^{H}$ is contractible.

Definition 2.2. Let $\Sigma$ be a subcomplex of $\Delta_{k}$ and let $x \in \Sigma$. Let $\Gamma$ be a group which acts on $\Delta_{k}$ by means of building automorphisms, [15], i.e., suppose there is a homomorphism $\Gamma \rightarrow$ Aut $\Delta_{k}$, where Aut $\Delta_{k}$ is the group of building automorphisms of $\Delta_{k}$. We say that $x$ is a $\Gamma$-centre of $\Sigma$ if $x$ is fixed by any element of $\Gamma$ that stabilizes $\Sigma$ setwise.

The following theorem is known as the "Centre Conjecture" of J. Tits, cf. [14, Lem. 1.2], [12, §4], [13, §2.4], [16], [9, Ch. 2, §3], [11, Conj. 3.3]. It has recently been proved in a series of intricate case-by-case arguments by B. Mühlherr and J. Tits [8] ( $G$ of classical type or type $\left.G_{2}\right)$, B. Leeb and C. Ramos-Cuevas [7] ( $G$ of type $F_{4}$ or $\left.E_{6}\right)$ and C. Ramos-Cuevas [10] ( $G$ of type $E_{7}$ or $E_{8}$ ).

Theorem 2.3 (Tits' Centre Conjecture). Let $\Sigma$ be a convex contractible subcomplex of $\Delta_{k}$. Then $\Sigma$ has an Aut $\Delta_{k}$-centre.

Remark 2.4. Suppose $G$ is semisimple and $k$ is a perfect field. It follows from [15, 5.7.2] that Aut $G$ is an algebraic group also defined over $k$. In [4, Thm. 5.31], we give a uniform proof of the following special case of the Centre Conjecture: Let $H$ be a subgroup of $G$. If $\Delta_{k}^{H}$ is contractible, i.e., if $\Delta_{k}^{H}$ is not $\Delta_{k}$-cr, then $\Delta_{k}^{H}$ admits an (Aut $\left.G\right)(k)$-centre. The proof of this result in [4] utilizes methods from geometric invariant theory and the concept of optimal destabilizing parabolic subgroups.

Let $k$ be a field, let $k_{s}$ denote its separable closure, and let $\bar{k}$ denote its algebraic closure. Note that $k_{s}=\bar{k}$ if $k$ is perfect. Thanks to [15, 5.7.2], $\Gamma:=\operatorname{Gal}\left(k_{s} / k\right)$ acts on $\Delta_{\bar{k}}$ via building automorphisms. In [4, Thm. 5.33], we show that if $H$ is a $k$-defined subgroup of $G$ such that $\Delta_{\bar{k}}^{H}$ is contractible, then $\Delta_{\bar{k}}^{H}$ admits a $\Gamma$-centre. The proof of [4, Thm. 5.33] rests on a rationality result concerning $G$-cr subgroups of $G$, [4, Prop. 5.14(iii)].

Both [4, Thm. 5.31] and [4, Thm. 5.33] improve on [2, Thm. 3.1]. 


\section{Proof of Theorem 1.1}

As noted above the reverse implication of Theorem 1.1 is proved in [4, Thm. 5.11]. We deduce the other direction with the aid of Theorem 2.3 .

Suppose $k_{1} / k$ is an algebraic separable extension of fields and let $\Delta_{k}$ and $\Delta_{k_{1}}$ denote the buildings of $G$ over $k$ and $k_{1}$, respectively. By the reverse implication, one may suppose that $k_{1} / k$ is Galois. Then the Galois group $\Gamma:=\operatorname{Gal}\left(k_{1} / k\right)$ acts simplicially on $\Delta_{k_{1}}$, i.e., $\Gamma$ permutes the set of $k_{1}$-defined parabolic subgroups of $G$. Moreover, the subcomplex of $\Delta_{k_{1}}$ consisting of $\Gamma$-stable simplices is just $\Delta_{k}$.

It is convenient to reduce to the case when $H$ is not contained in any $k$-defined Levi subgroup of any proper $k$-defined parabolic subgroup of $G$. To do this, we let $L$ be minimal such that $L$ is a $k$-defined Levi subgroup of some $k$-defined parabolic subgroup $P$ of $G$ and $H \subseteq L$. Then $L$ is also $k_{1}$-defined, and by a result of Serre, [13, Prop. 3.2], $H$ is $G$-completely reducible over $k$ (resp. $k_{1}$ ) if and only if $H$ is $L$-completely reducible over $k$ (resp. $k_{1}$ ). Now if $L^{\prime}$ is a $k$-defined Levi subgroup of some proper $k$-defined parabolic subgroup $Q$ of $L$, then $Q R_{u}(P)$ is a $k$-defined parabolic subgroup of $G$, and $L^{\prime}$ is a Levi subgroup of $Q R_{u}(P)$, 6, Prop. 4.4]. Since $L$ is minimal among those $k$-defined Levi subgroups of $k$-defined parabolic subgroups of $G$ that contain $H, H$ cannot be contained in $L^{\prime}$. By replacing $G$ with $L$, we can now assume that $H$ is not contained in any $k$-defined Levi subgroup of any proper $k$-defined parabolic subgroup of $G$.

Suppose that $H$ is not $G$-completely reducible over $k_{1}$. Then $\Delta_{k_{1}}^{H}$ is contractible, and since $H$ is $k$-defined, $\Delta_{k_{1}}^{H}$ is $\Gamma$-stable. Since $\Delta_{k_{1}}^{H}$ is a convex contractible subcomplex of $\Delta_{k_{1}}$, it follows from Theorem 2.3 that $\Gamma$ fixes a point of $\Delta_{k_{1}}^{H}$, and this point lies in some (minimal) simplex $\sigma_{P}$, where $P$ is a proper $k_{1}$-defined parabolic subgroup of $G$. Since the action of $\Gamma$ on $\Delta_{k_{1}}$ is simplicial, $P$ is stabilized by $\Gamma$, which is equivalent to saying that $P$ is $k$-defined. Now, by assumption, $H$ is not contained in any $k$-defined Levi subgroup of $P$, so $H$ is not $G$-completely reducible over $k$. This completes the proof of Theorem 1.1 .

Remark 3.1. In [5, Thm. 4.13], we prove a generalization of the reverse implication of Theorem 1.1 in the setting of "relative complete reducibility". The arguments above used to derive the forward direction of Theorem 1.1 do not apply to this more general situation, as the relevant subset in $\Delta_{k_{1}}$ is only a convex subset but not a subcomplex of $\Delta_{k_{1}}$. Thus Theorem 2.3 does not apply. 
Acknowledgements: The paper was written during a stay of the first and third authors at the Max Planck Institute for Mathematics in Bonn. The authors also acknowledge financial support from the DFGpriority program SPP1388 "Representation Theory", Marsden Grant UOC0501, and The Royal Society.

\section{REFERENCES}

[1] M. Bate, B. Martin, G. Röhrle, A geometric approach to complete reducibility, Invent. Math. 161, no. 1 (2005), 177-218.

[2] _ On Tits' Centre Conjecture for fixed point subcomplexes, C. R. Math. Acad. Sci. Paris 347 (2009), 353-356.

[3] M. Bate, B. Martin, G. Röhrle, R. Tange, Complete reducibility and separability, Trans. AMS, (2010), in press.

[4] Closed orbits and uniform S-instability in geometric invariant theory, preprint (2009) arXiv:0904.4853v3 [math.AG].

[5] - Complete reducibility and conjugacy classes of tuples in algebraic groups and Lie algebras, preprint (2009) arXiv:0905.0065v2 [math.GR].

[6] A. Borel, J. Tits, Groupes Réductifs, Inst. Hautes Études Sci. Publ. Math. 27 (1965), 55-150.

[7] B. Leeb, C. Ramos-Cuevas, The center conjecture for spherical buildings of types $F_{4}$ and $E_{6}$, Preprint. arXiv:0905.0839v1.

[8] B. Mühlherr, J. Tits, The Centre Conjecture for non-exceptional buildings, J. Algebra 300 (2006), no. 2, 687-706.

[9] D. Mumford, J. Fogarty, F. Kirwan, Geometric invariant theory. Third edition. Ergebnisse der Mathematik und ihrer Grenzgebiete, 34. Springer-Verlag, Berlin, 1994.

[10] C. Ramos-Cuevas, The center conjecture for thick spherical buildings, Preprint. arXiv:0909.2761 1 .

[11] G. Rousseau, Immeubles sphériques et théorie des invariants, C.R.A.S. 286 (1978), 247-250.

[12] J-P. Serre, La notion de complète réductibilité dans les immeubles sphériques et les groupes réductifs, Séminaire au Collège de France, résumé dans [17, pp. 93-98], (1997).

[13] _ Complète Réductibilité, Séminaire Bourbaki, 56ème année, 2003-2004, $\mathrm{n}^{\mathrm{o}} 932$.

[14] J. Tits, Groupes semi-simples isotropes, Colloq. Théorie des Groupes Algébriques, Bruxelles, 1962.

[15] _ Buildings of spherical type and finite BN-pairs, Lecture Notes in Math. 386, Springer-Verlag (1974).

[16] — Quelques cas d'existence d'un centre pour des ensembles de chambres qui sont convexes, non vides et ne contiennent pas de paires de chambres opposées, Séminaire au Collège de France, résumé dans [17, pp. 98-101], (1997).

[17] _ Théorie des groupes, Résumé des Cours et Travaux, Annuaire du Collège de France, $97^{\mathrm{e}}$ année, (1996-1997), 89-102. 
Department of Mathematics, University of York, York YO10 5DD, UNITED KINGDOM

E-mail address: meb505@york.ac.uk

Mathematics and Statistics Department, University of Canterbury, Private Bag 4800, Christchurch 8140, New Zealand

E-mail address: B.Martin@math.canterbury.ac.nz

Fakultät für Mathematik, Ruhr-Universität Bochum, 44780 Bochum, GERMANY

E-mail address: gerhard.roehrle@rub.de 